ANNALES

POLONICI MATHEMATICI

LVII.1 (1992)

\title{
A simulation of integral and derivative of the solution of a stochastic integral equation
}

\author{
by Nguyen Quy Hy and Nguyen Thi Minh (Hanoi)
}

\begin{abstract}
A stochastic integral equation corresponding to a probability space $\left(\Omega, \Sigma_{\omega}, P_{\omega}\right)$ is considered. This equation plays the role of a dynamical system in many problems of stochastic control with the control variable $u(\cdot): \mathbb{R}^{1} \rightarrow \mathbb{R}^{m}$. One constructs stochastic processes $\eta^{(1)}(t), \eta^{(2)}(t)$ connected with a Markov chain and with the space $\left(\Omega, \Sigma_{\omega}, P_{\omega}\right)$. The expected values of $\eta^{(i)}(t)(i=1,2)$ are respectively the expected value of an integral representation of a solution $x(t)$ of the equation and that of its derivative $x_{u}^{\prime}(t)$.
\end{abstract}

1. Introduction. Given a probability space $\left(\Omega, \Sigma_{\omega}, P_{\omega}\right)$. Let $L^{2}(\Omega)$ be the Hilbert space of all real random variables defined on $\left(\Omega, \Sigma_{\omega}, P_{\omega}\right)$ which have finite second moment:

$$
\begin{aligned}
L^{2}(\Omega)=\left\{\xi: \Omega \rightarrow \mathbb{R}^{1} \mid\|\xi\|_{L^{2}(\Omega)}:=\left[\int_{\Omega} \xi^{2}(\omega) P_{\omega}(d \omega)\right]^{1 / 2}\right. & \\
& \left.=\left[E_{\omega}\left\{\xi^{2}(\omega)\right\}\right]^{1 / 2}<\infty\right\} .
\end{aligned}
$$

We consider the stochastic equation

$$
x(t)=\int_{a}^{b} K(t, \tau) x(\tau) \mu(d \tau)+g(t, u(\cdot)) \quad(t \in\langle a, b\rangle(\bmod \mu))
$$

where $\langle a, b\rangle$ is a closed or open interval, $-\infty \leq a<b<\infty, \mu$ is Lebesgue measure on $\mathbb{R}^{1}, u(t) \in \mathbb{R}^{m}, x(t)=\left(x_{1}(t), \ldots, x_{n}(t)\right)^{T}, x_{i}(t) \in L^{2}(\langle a, b\rangle)$.

We suppose that the given functions in (1.2) satisfy the following conditions:

(A) The vector-valued function $u(t)=\left(u_{1}(t), \ldots, u_{m}(t)\right)^{T}:\langle a, b\rangle \rightarrow$ $(\underline{u}, \bar{u}) \subset \mathbb{R}^{m}$ is deterministic (where $(\underline{u}, \bar{u})=\left\{\left(u_{1}, \ldots, u_{m}\right): \underline{u}_{i}<\right.$ 
$\left.\left.u_{i}<\bar{u}_{i}, i=1, \ldots, m\right\}\right)$. The function $\bar{g}(t)=\left(\bar{g}_{1}(t), \ldots, \bar{g}_{n}(t)\right)^{T}:=$ $g(t, u(\cdot) ; \omega)$ is a Hilbert valued $n$-variate process with parameter $t \in$ $\langle a, b\rangle$, integrable on $(a, b)$ (see [8]).

(B) The matrix-valued function $K(t, \tau ; \omega):=\left(K_{i j}(t, \tau ; \omega)\right)_{n \times n}$ is a Hilbert valued $(n \times n)$-variate process with parameters $(t, \tau) \in\langle a, b\rangle \times$ $\langle a, b\rangle$.

Under some assumptions, there exists a unique solution of (1.2) which depends on $u(\cdot): x(t)=x(t, u(\cdot) ; \omega)$ and is a Hilbert valued $n$-variate process with parameter $t \in\langle a, b\rangle$, i.e. (see [8]) $x_{i}(t)=x_{i}(t, u(\cdot)) \in L^{2}(\Omega)$. In many general problems of stochastic control, we deal with the state equation of the form (1.2) (see [15], [5], [11], [12]) with $K(t, \tau)=0$ for $\tau>t \geq a ; x(t)$ is the state variable, $u(t)$ is the control variable.

Using gradient methods to solve the corresponding stochastic control problems, we must determine the random gradient $\Phi_{u}(x, u, t ; \omega)$ of some average cost $E\left\{\int_{a}^{b} \Phi d t\right\}$. This problem reduces to determining the expected value:

$$
\begin{aligned}
\bar{x}(t) & :=E_{\omega}\{x(t, u(\cdot) ; \omega)\}, \\
\bar{x}_{u}^{\prime}(t) & :=E_{\omega}\left\{x_{u}^{\prime}(t, u(\cdot) ; \omega)\right\},
\end{aligned}
$$

where the components of the matrix $x_{u}^{\prime}$ :

$$
\frac{\partial x_{i}(t, u(\cdot) ; \omega)}{\partial u_{j}}=\left.\frac{\partial x_{i}(t, u ; \omega)}{\partial u_{j}}\right|_{u_{j}=u_{j}(\cdot)}
$$

are the mean quadratic derivatives (m.q.d.) of the stochastic process $x_{i}\left(t, u_{1}, \ldots, u_{m} ; \omega\right)$ with respect to the parameter $u_{j}$ (see [8] or (3.1b)). Further, we have to determine the following vector and matrix:

$$
\begin{aligned}
\left\langle\varphi^{(1)}, x\right\rangle & :=E_{\omega}\left\{\int_{a}^{b} \varphi^{(1)}(t ; \omega) x(t, u(\cdot) ; \omega) \mu(d t)\right\}, \\
\left\langle\varphi^{(2)}, x_{u}^{\prime}\right\rangle: & =E_{\omega}\left\{\int_{a}^{b} \varphi^{(2)}(t ; \omega) x_{u}^{\prime}(t, u(\cdot) ; \omega) \mu(d t)\right\} .
\end{aligned}
$$

Here $\varphi^{(i)}(i=1,2)$ satisfy the following condition:

$\left(\mathrm{C}_{i}\right) \quad \varphi^{(i)}(t ; \omega)$ is a Hilbert valued $\left(n_{i} \times n\right)$-variate process with components having second moment integrable on $\langle a, b\rangle$.

When $n_{1}=n$ and $\varphi^{(1)}(t ; \omega)$ is the unit matrix of order $n$, problem (1.5) reduces to determining the expected value of the integral of the solution of (1.2) on $\langle a, b\rangle$. We can also investigate the similar problem for the integral of the derivatives of the solution. 
In particular, when $n=m=1, u(t)=t$, and $K(t, \tau)$ and $g(t, u(\cdot))=$ $g(u(t))=g(t)$ are deterministic, (1.2) takes the form of a Fredholm equation of the second type in $L^{2}(a, b)$. Then an unbiased estimator (u.e.) and an asymptotic u.e. of (1.3), (1.5) are obtained in [14], [10], [9].

When $g(t)=E_{\omega}\{\eta(t ; \omega)\}$ and $K(t, \tau)=E_{\omega}\{\chi(t, \tau ; \omega)\}$, analogous estimators are constructed basing on a realization of the processes $\eta^{(t)}, \chi(t, \tau)$ (see [7], [6], [4], [1]). An asymptotic u.e. and an u.e. of (1.4), (1.5) are also constructed for $\chi(t, \tau) \geq 0$ (see $[3]$ ).

In this paper, we provide an u.e. of (1.3)-(1.6) for the stochastic integral equation (1.2).

By the above method we can apply the Monte Carlo method to the numerical calculation of the quantities (1.3)-(1.6).

\section{A random model connected with an integral transformation} of a solution. Let $L_{1 \times 1}^{2}=L_{1 \times 1}^{2}(\Omega \times(a, b))$ be the class of Hilbert valued processes with second moment integrable on $\langle a, b\rangle$ :

$$
\text { (2.1) } L_{1 \times 1}^{2}=\left\{f:\langle a, b\rangle \rightarrow L^{2}(\Omega) \mid\|f\|_{L_{1 \times 1}^{2}}^{2}=\int_{a}^{b} E_{\omega}\left\{f^{2}(t)\right\} \mu(d t)<\infty\right\} .
$$

Then $L_{1 \times 1}^{2}$ is a Hilbert space (see [2]).

Let $L_{n \times s}^{2}=L_{n \times s}^{2}(\Omega \times(a, b))$ be the class of Hilbert valued $(n \times s)$-variate processes with components having second moment integrable on $(a, b)$ :

$$
\begin{aligned}
& L_{n \times s}^{2}=\left\{F=\left(F_{i j}\right)_{n \times s} \mid F_{i j}:\langle a, b\rangle \rightarrow L^{2}(\Omega),\right. \\
& \left.\quad \int_{a}^{b}\left\|F_{i j}(t)\right\|_{L^{2}(\Omega)}^{2} \mu(d t)=\int_{a}^{b} \int_{\Omega} F_{i j}^{2}(t ; \omega) P_{\omega}(d \omega) \mu(d t)<\infty\right\} .
\end{aligned}
$$

We can represent $L_{n \times s}^{2}$ in the form $L_{n \times s}^{2}=L_{1 \times 1}^{2} \oplus \ldots \oplus L_{1 \times 1}^{2}(n \times s$ summands). Hence $L_{n \times s}^{2}$ is also a Hilbert space (see [2]) with the norm

$$
\|F\|_{L_{n \times s}^{2}}=\left(\sum_{i=1}^{n} \sum_{j=1}^{s}\left\|F_{i j}\right\|_{L_{n \times s}^{2}}^{2}\right)^{1 / 2} \quad\left(\forall F \in L_{n \times s}^{2}\right) .
$$

Let $\mathcal{K}$ and $\mathcal{K}_{+}$be the following integral operators:

$$
\begin{aligned}
{[\mathcal{K} f](t) } & =\int_{a}^{b} K(t, \tau) f(\tau) \mu(d \tau) \quad\left(f \in L_{n \times 1}^{2}\right), \\
{\left[\mathcal{K}_{+} f\right](t) } & =\int_{a}^{b} K_{+}(t, \tau) f(\tau) \mu(d \tau) \quad\left(f \in L_{n \times 1}^{2}\right),
\end{aligned}
$$

where for each matrix $H=\left(H_{i j}\right)_{\bar{n} \times \bar{m}}$ we write $H_{+}=\left(\mid H_{i j}\right)_{\bar{n} \times \bar{m}}$. We suppose that: 
Then we have (see [8])

$$
P_{\theta}\left(\bigcup_{k=0}^{\infty} \Theta_{k}\right)=1
$$

It follows from (2.10), (2.12) that $\bigcup_{k=0}^{\infty} \Theta_{k} \subset \Theta$ and from (2.13) we obtain

$$
P_{\theta}\left(\Theta \backslash \bigcup_{k=0}^{\infty} \Theta_{k}\right)=0 \text {. }
$$

Moreover, from (2.12), it is easy to see that

$$
\Theta_{k} \cap \Theta_{k^{\prime}}=\emptyset \quad\left(\forall k \neq k^{\prime}\right)
$$

Notice that the space

$$
\left(\Omega^{\infty}, \Sigma_{\bar{\omega}}, P_{\bar{\omega}}\right):=\left(\prod_{i=0}^{\infty} \Omega, \bigotimes_{i=0}^{\infty} \Sigma_{\omega} ; \prod_{i=0}^{\infty} P_{\omega}\right)
$$

is a probability space. Its elementary events take the form $\bar{\omega}:=\left(\omega_{0}, \ldots\right.$, $\left.\omega_{k}, \ldots\right), \omega_{i} \in \Omega(i=0,1,2, \ldots)$. Consider the product probability space $\left(\Theta \times \Omega^{\infty}, \Sigma_{\theta, \bar{\omega}}, P_{\theta, \bar{\omega}}\right)$ where

$$
\begin{array}{cc}
\Sigma_{\theta, \bar{\omega}}=\Sigma_{\theta} \otimes \Sigma_{\bar{\omega}}, & P_{\theta, \bar{\omega}}=P_{\theta} \times P_{\bar{\omega}}, \\
\Sigma_{\bar{\omega}}=\bigotimes_{i=0}^{\infty} \Sigma_{\omega}, & P_{\bar{\omega}}=\prod_{i=0}^{\infty} P_{\omega}
\end{array}
$$

and a mapping $\eta^{(1)}: \Theta \times \Omega \rightarrow \mathbb{R}^{n_{1}}$ defined by

$$
\begin{aligned}
& \eta^{(1)}(\theta ; \bar{\omega})=F^{(1)}\left(\theta, \bar{\omega} ; \varphi^{(1)}, \bar{g}\right) \\
& :=\sum_{k=0}^{\infty} \mathbf{1}_{\Theta_{k}}(\theta) \frac{\varphi^{(1)}\left(\theta_{0} ; \omega_{0}\right)}{p_{0}\left(\theta_{0}\right) q\left(\theta_{k}\right)} \prod_{i=0}^{k} \frac{K\left(\theta_{i-1}, \theta_{i} ; \omega_{i-1}\right)}{p\left(\theta_{i-1}, \theta_{i}\right)} \bar{g}\left(\theta_{k} ; \omega_{k}\right) \\
& \quad((\theta, \bar{\omega}) \in \Theta \times \Omega),
\end{aligned}
$$

with the convention that

$$
\frac{K\left(\theta_{-1}, \theta_{0} ; \omega_{-1}\right)}{p\left(\theta_{-1}, \theta_{0}\right)}:=1,
$$

The measurability of $\varphi^{(1)}, K, g$ (see assumptions $\left.\left(\mathrm{C}_{1}\right),(\mathrm{B}),\left(\mathrm{B}_{1}\right),(\mathrm{A})\right)$ and (2.5), (2.7), (2.17) yield the $\Sigma_{\theta, \bar{\omega}}$-measurability of $\eta^{(1)}(\theta ; \bar{\omega})$ on $\Theta \times \Omega^{\infty}$, i.e. $\eta^{(1)}(\theta ; \bar{\omega})$ is a random $n_{1}$-variate vector defined on $\left(\Theta \times \Omega^{\infty}, \Sigma_{\theta, \bar{\omega}}, P_{\theta, \bar{\omega}}\right)$. We suppose that the random processes $\varphi(t ; \omega), d(t ; \omega)$ fulfil the following condition:

$\left(\mathrm{D}^{\prime}\right) \quad$ For given $\left(\theta_{0}, \ldots, \theta_{k}\right)$, the random variables ${ }^{\prime} \varphi\left(\theta_{0} ; \omega\right), K\left(\theta_{j-1}, \theta_{j} ; \omega\right)$ $(j=1, \ldots, k), d\left(\theta_{k} ; \omega\right)$ are mutually independent $(\forall k=1,2, \ldots)$. 
Now we put

(2.24) $\quad \eta_{i}(k ; \theta, \bar{\omega}):=\sum_{j=1}^{n}\left[\frac{\varphi^{(1)}\left(\theta_{0} ; \omega_{0}\right)}{p_{0}\left(\theta_{0}\right) q\left(\theta_{k}\right)} \prod_{l=0}^{k} \frac{K\left(\theta_{l-1}, \theta_{l} ; \omega_{l-1}\right)}{p\left(\theta_{l-1}, \theta_{l}\right)}\right]_{i j} \bar{g}_{j}\left(\theta_{k} ; \omega_{k}\right)$

$$
(i=1, \ldots, n)
$$

where for each matrix $A=\left(a_{i j}\right)$ we write $[A]_{i j}=a_{i j}$. Then from Fubini's theorem it follows that

$$
\begin{aligned}
Y_{i}(k):= & \int_{\theta_{k} \times \Omega^{\infty}}\left|\eta_{i}(k ; \theta, \bar{\omega})\right| P_{\theta, \bar{\omega}}(d \theta \times d \bar{\omega}) \\
\leq & \sum_{j=1}^{n} \int_{\Theta_{k} \times \Omega^{\infty}} \mid\left[\frac{\varphi^{(1)}\left(\theta_{0} ; \omega_{0}\right)}{p_{0}\left(\theta_{0}\right) q\left(\theta_{k}\right)}\right. \\
& \left.\times \prod_{l=0}^{k} \frac{K\left(\theta_{l-1}, \theta_{l} ; \omega_{l-1}\right)}{p\left(\theta_{l-1}, \theta_{l}\right)}\right]_{i j} \bar{g}_{j}\left(\theta_{k} ; \omega_{k}\right) P_{\theta}(d \theta) P_{\bar{\omega}}(d \bar{\omega}) .
\end{aligned}
$$

From (D), (2.25), (2.8)-(2.11), (2.19) we have (with $(k+1)$-fold integration)

$$
\begin{aligned}
& Y_{i}(k) \leq E_{\omega}\left\{\int_{a}^{b}\right. \ldots \int_{a}^{b} \sum_{j=1}^{n} \mid\left[\varphi^{(1)}\left(\theta_{0} ; \omega\right)\right. \\
&\left.\left.\times \prod_{l=0}^{k} K\left(\theta_{l-1}, \theta_{l} ; \omega\right)\right]_{i j} \bar{g}_{j}\left(\theta_{k} ; \omega\right) \mid \mu\left(d \theta_{0}\right) \ldots \mu\left(d \theta_{k}\right)\right\} \\
& \leq E_{\omega}\left\{\left[\int_{a}^{b} \ldots \int_{a}^{b} \varphi_{+}^{(1)}\left(\theta_{0} ; \omega\right)\right.\right. \\
&\left.\left.\quad \times \prod_{l=0}^{k} K_{+}\left(\theta_{l-1}, \theta_{l} ; \omega\right) \bar{g}_{+}\left(\theta_{k} ; \omega\right)\right]_{i} \mu\left(d \theta_{0}\right) \ldots \mu\left(d \theta_{k}\right)\right\}
\end{aligned}
$$

Therefore (see (2.4))

$$
Y_{i}(k) \leq E_{\omega}\left\{\int_{a}^{b}\left[\varphi_{+}^{(1)}\left(\theta_{0} ; \omega\right) \mathcal{K}_{+}^{k} \bar{g}_{+}\left(\theta_{0} ; \omega\right)\right]_{i} \mu\left(d \theta_{0}\right)\right\} .
$$

Using Hölder's inequality, by $(2.25),(2.27),\left(2.2^{\prime}\right)$ and $\left(\mathrm{C}_{1}\right),\left(\mathrm{B}_{1}\right),(\mathrm{A})$ we obtain

$$
\begin{aligned}
& \int_{\Theta_{k} \times \Omega^{\infty}}\left|\eta_{i}(k ; \theta, \bar{\omega})\right| P_{\theta, \bar{\omega}}(d \theta \times d \bar{\omega}) \\
& \leq\left\|\varphi_{+}^{(1)}\right\|_{L_{n_{1} \times n}^{2}}\left\|\mathcal{K}_{+}^{k} \bar{g}_{+}\right\|_{L_{n \times 1}^{2}}<\infty .
\end{aligned}
$$


TheOREM 1. Suppose that assumptions $(\mathrm{A}),(\mathrm{B}),\left(\mathrm{B}_{1}\right),\left(\mathrm{C}_{1}\right)$ are satisfied and $\varphi(t)=\varphi^{(1)}(t ; \omega)$ and $d(t)=\bar{g}(t ; \omega)$ fulfil condition (D). Then the equation (1.2) has a solution $x(t)=x(t, u(\cdot) ; \omega)$ in $L_{n \times 1}^{2}$. Moreover, the expected value $E_{\theta, \bar{\omega}}\left\{\eta^{(1)}(\theta ; \bar{\omega})\right\}$ exists and is finite and

$$
M_{\theta, \bar{\omega}}\left\{\eta^{(1)}(\theta ; \bar{\omega})\right\}=\left\langle\varphi^{(1)}, x\right\rangle .
$$

Proof. For each vector $h=\left(h_{1}, \ldots, h_{n}\right)^{T} \in \mathbb{R}^{n}$, we set $[h]_{i}:=h_{i}$. Therefore, from $\left(B_{1}\right)$, it follows that

$$
\begin{aligned}
\mid \int_{a}^{b}[K(t, \tau ; \omega) f(\tau ; \omega)]_{i} & \mu(d \tau) \mid \\
& \leq \int_{a}^{b}\left[K_{+}(t, \tau ; \omega) f_{+}(\tau ; \omega)\right]_{i} \mu(d \tau)<\infty
\end{aligned}
$$

$i=1, \ldots, n),\left(\forall f \in L_{n \times 1}^{2}, t \in\langle a, b\rangle(\bmod \mu), \omega \in \Omega\left(\bmod P_{\omega}\right)\right)$; further, from (2.2)-(2.4) we have

$$
\begin{aligned}
\|\mathcal{K} f\|_{L_{n \times 1}^{2}} & \leq\left\|\mathcal{K}_{+} f_{+}\right\|_{L_{n \times 1}^{2}} \leq\left\|\mathcal{K}_{+}\right\|\left\|f_{+}\right\|_{L_{n \times 1}^{2}} \\
& =\left\|\mathcal{K}_{+}\right\|\|f\|_{L_{n \times 1}^{2}} \quad\left(\forall f \in L_{n \times 1}^{2}\right),
\end{aligned}
$$

i.e. the operator $\mathcal{K}: L_{n \times 1}^{2} \rightarrow L_{n \times 1}^{2}$ is bounded. Put

$$
\begin{array}{ll}
\mathcal{S}^{(k)}:=\sum_{j=0}^{k} \mathcal{K}^{j} \bar{g}=\bar{g}+\mathcal{K} \mathcal{S}^{(k-1)}, & \mathcal{S}^{(0)}=\bar{g} \\
\mathcal{S}_{+}^{(k)}:=\sum_{j=0}^{k} \mathcal{K}_{+}^{j} \bar{g}_{+}=\bar{g}_{+}+\mathcal{K}_{+} \mathcal{S}_{+}^{(k-1)}, & \mathcal{S}_{+}^{(0)}=\bar{g}_{+} .
\end{array}
$$

Then for some natural number $p$, it follows from (2.2)-(2.4) and (2.19) that

$$
\left\|\mathcal{S}^{(k+p)}-\mathcal{S}^{(k)}\right\|_{L_{n \times 1}^{2}} \leq\left\|\mathcal{S}_{+}^{(k+p)}-\mathcal{S}_{+}^{(k)}\right\|_{L_{n \times 1}^{2}} \quad(\forall k=1,2, \ldots) .
$$

As the Neumann series $\sum_{j=0}^{\infty} \mathcal{K}_{+}^{j} \bar{g}_{+}=$l.i.m. $k \rightarrow \infty \mathcal{S}_{+}^{(k)}$ converges in $L_{n \times 1}^{2}$ (by $\left.(\mathrm{A}),\left(\mathrm{B}_{1}\right)\right),\left\{\mathcal{S}_{+}^{(k)}\right\}_{k}$ is a Cauchy sequence. Hence, by $(2.22)$ we deduce the convergence of $\sum_{j=0}^{\infty} \mathcal{K}^{j} \bar{g}=1$. i.m. $k \rightarrow \infty \mathcal{S}^{(k)}$ in the Hilbert space $L_{n \times 1}^{2}$. Therefore from the continuity of $\mathcal{K}$, we have

$$
x=\sum_{j=0}^{\infty} \mathcal{K}^{j} \bar{g}
$$

( $x$ is a solution in $L_{n \times 1}^{2}$ of $(1.2)$ ). 
From (2.17), (2.24), (2.27) and $\left(\mathrm{B}_{1}\right),\left(\mathrm{C}_{1}\right)$ we have

$$
\begin{aligned}
& \sum_{k=0}^{\infty} \quad \int_{\Theta_{k} \times \Omega^{\infty}}\left|\left[\eta^{(1)}(\theta ; \bar{\omega})\right]_{i}\right| P_{\theta, \bar{\omega}}(d \theta \times d \bar{\omega}) \\
& \quad=\sum_{k=0}^{\infty} \int_{\Theta_{k} \times \Omega^{\infty}}\left|\eta_{i}(k ; \theta, \bar{\omega})\right| P_{\theta, \bar{\omega}}(d \theta \times d \bar{\omega})=\sum_{k=0}^{\infty} Y_{i}(k) \\
& \quad \leq \int_{\Omega} \int_{a}^{b}\left[\varphi_{+}^{(1)}\left(\theta_{0} ; \omega\right) \sum_{k=0}^{\infty}\left(\mathcal{K}_{+}^{k} \bar{g}_{+}\right)\left(\theta_{0} ; \omega\right)\right]_{i} \mu\left(d \theta_{0}\right) P_{\omega}(d \omega)<\infty .
\end{aligned}
$$

Hence, by $(2.14),(2.17),(2.24),(2.28)$ it is easy to deduce the existence and finiteness of the expected value

$$
\text { (2.30) } \quad E_{\theta, \bar{\omega}}\left\{\left[\eta^{(1)}(\theta ; \bar{\omega})\right]_{i}\right\}=\sum_{k=0}^{\infty} \int_{\Theta_{k} \times \Omega^{\infty}} \eta_{i}(k ; \theta, \bar{\omega}) P_{\theta, \bar{\omega}}(d \theta \times d \omega)<\infty .
$$

Then, using Fubini's theorem, from the convergence of the series (2.29) and from $(2.23),(1.5)$, we get

$$
\begin{aligned}
E_{\theta, \bar{\omega}}\left\{\left[\eta^{(1)}(\theta ; \bar{\omega})\right]_{i}\right\} & =E_{\omega}\left\{\left[\int_{a}^{b} \varphi^{(1)}\left(\theta_{0} ; \omega\right) \sum_{k=0}^{\infty} \mathcal{K}^{k} \bar{g}\left(\theta_{0} ; \omega\right) \mu\left(d \theta_{0}\right)\right]_{i}\right\} \\
& =E_{\omega}\left\{\left[\int_{a}^{b} \varphi^{(1)}\left(\theta_{0} ; \omega\right) x\left(\theta_{0} ; \omega\right) \mu\left(d \theta_{0}\right)\right]_{i}\right\} \\
& =\left[\left\langle\varphi^{(1)}, x\right\rangle\right]_{i} \quad\left(i=1, \ldots, n_{1}\right) .
\end{aligned}
$$

This completes the proof.

Corollary 1. Suppose assumptions (A), (B), $\left(\mathrm{B}_{1}\right)$ are satisfied and $\varphi(t)=K\left(t_{0}, t ; \omega\right)\left(\forall t_{0} \in(a, b)\right)$ and $d(t)=\bar{g}(t ; \omega)$ fulfil condition (D). Then the expected value $E_{\theta, \bar{\omega}}\left\{\xi^{(1)}\left(t_{0} ; \theta, \omega\right)\right\}$ exists and is finite and

$$
E_{\theta, \bar{\omega}}\left\{\xi^{(1)}\left(t_{0} ; \theta, \bar{\omega}\right)\right\}=\bar{x}\left(t_{0}\right) \quad\left(\forall t_{0} \in\langle a, b\rangle(\bmod \mu)\right),
$$

where $x(t)=x(t, u(\cdot) ; \omega)$ is a solution of $(1.2)$ and

$$
\xi^{(1)}\left(t_{0} ; \theta, \bar{\omega}\right)=F^{(1)}\left(\theta, \bar{\omega} ; K\left(t_{0}, \cdot\right), \bar{g}\right)+\bar{g}\left(t_{0} ; \omega_{0}\right) \text {. }
$$

Proof. From $(2.20),\left(2.2^{\prime}\right)$, it is easy to deduce

$$
\int_{a}^{b} \int_{\Omega}\left(\int_{a}^{b} K_{i j}\left(t_{0}, \tau ; \omega\right) f(\tau ; \omega) \mu(d \tau)\right)^{2} P_{\omega}(d \omega) \mu(d t)<\infty
$$

$$
\left(\forall t_{0} \in\langle a, b\rangle(\bmod \mu) ; \forall f(\cdot) \in L_{n \times 1}^{2}\right) .
$$


Then (see p. 380 of [2]) for a fixed $t_{0} \in\langle a, b\rangle$, we easily see that $\varphi^{(1)}(t ; \omega)=$ $K\left(t_{0}, t ; \omega\right)$ satisfies condition $\left(\mathrm{C}_{1}\right)$. By Theorem 1 , we have

$$
\begin{aligned}
\left\langle K\left(t_{0}, \cdot\right), x\right\rangle=E_{\theta, \bar{\omega}} & \left\{F^{(1)}\left(\theta, \bar{\omega} ; K\left(t_{0}, \cdot\right), \bar{g}\right)\right\} \\
& \left(\forall t_{0} \in\langle a, b\rangle(\bmod \mu) \quad(\text { see }(2.17),(2.18))\right)
\end{aligned}
$$

Further, since $x(t)$ is a solution of (1.2), therefore we have

$$
E_{\omega}\left\{x\left(t_{0}\right)\right\}=\left\langle K\left(t_{0}, \cdot\right), x\right\rangle+E_{\omega}\left\{\bar{g}\left(t_{0} ; \omega\right)\right\}\left(\forall t_{0} \in\langle a, b\rangle(\bmod \mu)\right) .
$$

But $E_{\omega}\left\{\bar{g}\left(t_{0} ; \omega\right)\right\}=E_{\theta, \bar{\omega}}\left\{\bar{g}\left(t_{0} ; \omega_{0}\right)\right\}$. Hence, from (2.34), (2.33), (1.3) we deduce (2.31).

This completes the proof.

\section{A random model connected with an integral transformation} of the derivative of the solution. We consider a Hilbert valued $n$-variate process with parameters $(t, u)=\left(t, u_{1}, \ldots, n_{m}\right)^{T}$ having the m.q.d. with respect to the parameters $u=\left(u_{1}, \ldots, u_{m}\right)^{T} \in(\underline{u}, \bar{u})$ :

$$
\begin{aligned}
f_{u}^{\prime}(t, u) & =\left(f_{u_{1}}^{\prime}(t, u), \ldots, f_{u_{m}}^{\prime}(t, u)\right), \quad \text { where } \\
f_{u_{j}}^{\prime}(t, u) & =\left(\partial f_{1}(t, u) / \partial u_{j}, \ldots, \partial f_{n}(t, u) / \partial u_{j}\right)^{T} .
\end{aligned}
$$

Here, $\partial f_{i}(t, u) / \partial u_{j}$ is the m.q.d. of the Hilbert valued (scalar) process $f_{i}\left(t, u_{1}, \ldots, u_{m}\right)$ with parameter $u_{j} \in\left(\underline{u}_{j}, \bar{u}_{j}\right)$ (see [8]). Now, we set

$$
f_{u}^{\prime}(t, u(\cdot) ; \omega)=\left.f_{u}^{\prime}(t, u ; \omega)\right|_{u=u(\cdot)}
$$

and consider the equation

$$
x(t, u)=\int_{a}^{b} K(t, \tau) x(\tau, u) \mu(d \tau)+g(t, u)
$$

$(\forall t \in\langle a, b\rangle(\bmod \mu), \forall u \in(\underline{u}, \bar{u}))$.

Suppose that:

( $\left.\mathrm{A}_{1}\right) \quad g(t, u)$ is a Hilbert valued $n$-variate process with parameters $(t, u)$ $\in\langle a, b\rangle \times(\underline{u}, \bar{u})$ and $g(\cdot, u) \in L_{n \times 1}^{2}(\forall u \in(\underline{u}, \bar{u}))$.

$\left(\mathrm{A}_{2}\right) \quad$ For a fixed $t \in\langle a, b\rangle$, the stochastic process $g(t, u)=\left(g_{1}(t, u), \ldots\right.$ $\left.\ldots, g_{n}(t, u)\right)^{T}$ has a m.q.d. with respect to the parameters $u=$ $\left(u_{1}, \ldots, u_{m}\right) \in(\underline{u}, \bar{u})$ so that

$$
\int_{a}^{b} E_{\omega}\left\{\partial g_{i}(t, u(\cdot) ; \omega) / \partial u_{j}\right\} \mu(d t)<\infty .
$$

Moreover, for a fixed $t \in\langle a, b\rangle, \partial g_{i}(t, u) / \partial u_{j}(i=1, \ldots, n, j=$ $1, \ldots, m)$ are Hilbert valued processes, mean quadratic continuous in $u \in(\underline{u}, \bar{u})$. 
$\left(\mathrm{B}_{2}\right) \quad$ The inverse operator $(I-\mathcal{K})^{-1}: L_{n \times 1}^{2} \rightarrow L_{n \times 1}^{2}$ exists and is bounded, where $I: L_{n \times 1}^{2} \rightarrow L_{n \times 1}^{2}$ is the identity operator.

From assumptions $\left(\mathrm{A}_{2}\right),\left(\mathrm{B}_{2}\right),\left(\mathrm{C}_{2}\right)$, we can construct a random $\left(n_{2} \times\right.$ $m)$-variate $\eta^{(2)}(\theta ; \bar{\omega})$ defined on $\left(\Theta \times \Omega^{\infty}, \Sigma_{\theta, \bar{\omega}}, P_{\theta, \bar{\omega}}\right)$ like $\eta^{(1)}(\theta ; \bar{\omega})$ :

$$
\begin{aligned}
& \eta^{(2)}(\theta ; \bar{\omega})=F^{(2)}\left(\theta, \bar{\omega} ; \varphi^{(2)}\right) \\
& =\sum_{k=0}^{\infty} \mathbf{1}_{\Theta_{k}}(\theta) \frac{\varphi^{(2)}\left(\theta_{0} ; \omega_{0}\right)}{p_{0}\left(\theta_{0}\right) q\left(\theta_{k}\right)} \prod_{i=0}^{k} \frac{K\left(\theta_{i-1}, \theta_{i} ; \omega_{i-1}\right)}{p\left(\theta_{i-1}, \theta_{i}\right)} g_{u}\left(\theta_{k}, u(\cdot) ; \omega_{0}\right) .
\end{aligned}
$$

THEOREM 2. Suppose that assumptions $\left(\mathrm{A}_{1}\right),\left(\mathrm{A}_{2}\right),(\mathrm{B}),\left(\mathrm{B}_{1}\right),\left(\mathrm{B}_{2}\right),\left(\mathrm{C}_{2}\right)$ are satisfied and $\varphi(t)=\varphi^{(2)}(t ; \omega), d(t)=g_{u}^{\prime}(t, u(\cdot) ; \omega)$ fulfil condition (D). Then:

(i) For all $u \in(\underline{u}, \bar{u})$ the equation (3.2) has a unique solution and $x(\cdot, u) \in L_{n \times 1}^{2}$.

(ii) For all $(t, u) \in\langle a, b\rangle \times(\underline{u}, \bar{u})$, the m.q.d. $x_{u}^{\prime}(t, u)$ exists such that $x_{u}^{\prime}(\cdot, u) \in L_{n_{2} \times m}^{2}$.

(iii) The expected value of the random $\left(n_{2} \times n\right)$-variate vector $\eta^{(2)}(\theta ; \omega)$ exists and is finite and we have

$$
E_{\theta, \bar{\omega}}\left\{\eta^{(2)}(\theta ; \bar{\omega})\right\}=\left\langle\varphi^{(2)}, x_{u}^{\prime}\right\rangle
$$

P r o of. From $\left(\mathrm{B}_{1}\right),\left(\mathrm{A}_{1}\right)$ it follows that the solution $x(t, u)$ of $(3.2)$ exists and is represented by a formula like (2.23). Hence, by $\left(B_{2}\right)$ we can write it uniquely as

$$
x(t, u)=\left[(I-\mathcal{K})^{-1} g(\cdot, u)\right](t) \quad(\forall u \in(\underline{u}, \bar{u})) .
$$

The conclusion (i) is proved.

Since $(I-\mathcal{K})^{-1}$ is linear and bounded, from $\left(\mathrm{A}_{2}\right),(3.5)$ we deduce (see [13]) that the m.q.d. $x_{u}^{\prime}(t, u)$ exists and

$$
\begin{array}{r}
x_{u_{j}}^{\prime}(t, u)=\left[(I-\mathcal{K})^{-1} g_{u_{j}}^{\prime}(\cdot ; u)\right](t)=\left[\sum_{n=0}^{\infty} \mathcal{K}^{(n)} g_{u_{j}}^{\prime}(\cdot ; u)\right](t) \\
(j=1, \ldots, m) .
\end{array}
$$

Hence, from $\left(\mathrm{A}_{2}\right),\left(\mathrm{B}_{2}\right)$ we get (ii). By (3.6), it is easy to see that $x_{u}^{\prime}(t, u(\cdot))$ is the solution of the equation

$$
x_{u_{j}}^{\prime}(t, u(\cdot))=\int_{a}^{b} K(t, \tau) x_{u_{j}}^{\prime}(\tau, u(\cdot)) \mu(d \tau)+g_{u_{j}}^{\prime}(t, u(\cdot)) .
$$

From $\left(A_{2}\right),(B),\left(B_{1}\right),\left(C_{2}\right),(D)$ it follows that the assumptions of Theorem 1 are satisfied for (3.7). 
Then the random vector

$$
\begin{array}{r}
\left(\left[\eta^{(2)}(\theta ; \bar{\omega})\right]_{1 j}, \ldots,\left[\eta^{(2)}(\theta ; \bar{\omega})\right]_{n_{2} j}\right)^{T}=F^{(1)}\left(\theta, \bar{\omega} ; \varphi^{(2)}, g_{u_{j}}^{\prime}\right) \\
\quad(j=1, \ldots, m),
\end{array}
$$

has finite expected value, and (see (2.18), (3.1), (1.6), (3.3))

$$
E_{\bar{\omega}}\left\{\left[\eta^{(2)}(\theta ; \bar{\omega})\right]_{i j}\right\}=\left[\left\langle\varphi^{(2)}, x_{u_{j}}^{\prime}\right\rangle\right]_{i}=\left[\left\langle\varphi^{(2)}, x_{u}^{\prime}\right\rangle\right]_{i j} \quad\left(i=1, \ldots, n_{2}\right),
$$

i.e. we get (iii).

This completes the proof.

By Theorem 2, we can deduce the following result like in the proof of Corollary 1:

Corollary 2. Suppose that assumptions $\left(\mathrm{A}_{1}\right),\left(\mathrm{A}_{2}\right),(\mathrm{B}),\left(\mathrm{B}_{1}\right),\left(\mathrm{B}_{2}\right)$ are satisfied, and $\varphi(t)=K\left(t_{0}, t ; \omega\right)\left(\forall t_{0} \in\langle a, b\rangle\right), d(t)=g_{u}^{\prime}(t, u(\cdot) ; \omega)$ fulfil condition (D). Then the expected value $E_{\theta, \bar{\omega}}\left\{\xi^{(2)}\left(t_{0} ; \theta, \bar{\omega}\right)\right\}$ exists and is finite and $E_{\theta, \bar{\omega}}\left\{\xi^{(2)}\left(t_{0} ; \theta, \bar{\omega}\right)\right\}=E_{\omega}\left\{x_{u}^{\prime}\left(t_{0}\right)\right\}=\bar{x}_{u}^{\prime}\left(t_{0}\right)(\forall t \in\langle a, b\rangle(\bmod \mu))$, where $\xi^{(2)}\left(t_{0} ; \theta, \bar{\omega}\right)=F^{(2)}\left(\theta, \bar{\omega} ; K\left(t_{0}, \cdot\right)\right)+g_{u}^{\prime}\left(t, u(\cdot) ; \omega_{0}\right)$.

Rem ark. From (2.19), from Schwarz's and Hölder's inequalities and from Banach's theorem (see p. 159 of [13]) it is easy to see that $(B),\left(B_{1}\right)$, $\left(\mathrm{B}_{2}\right)$ can be replaced by the following assumption:

$\left(\mathrm{B}^{*}\right) \quad$ There exists a natural number $l$ so that

$$
K^{(l)}:=\operatorname{vraisup}\left\{\int_{a}^{b} \ldots \int_{a}^{b} \prod_{i=1}^{l}\left\|K\left(t_{i-1}, t_{i}\right)\right\|_{\mathbb{R}^{n \times n}}^{2} \mu\left(d t_{0}\right) \ldots \mu\left(d t_{l}\right)\right\}<1
$$

(with $l+1$ integrals),

$$
K^{(1)}:=\operatorname{vraisup}\left\{\int_{a}^{b} \int_{a}^{b}\|K(t, \tau ; \omega)\|_{\mathbb{R}^{n \times n}}^{2} \mu(d \tau) \mu(d t)\right\}<\infty,
$$

where

$$
\|K(t, \tau ; \omega)\|_{\mathbb{R}^{n \times n}}^{2}=\left(\sum_{i=1}^{n} \sum_{j=1}^{n}[K(t, \tau ; \omega)]_{i j}^{2}\right)^{1 / 2}
$$

\section{References}

[1] Nguyen Ngoc Cuong, On a solution of a class of random integral equations relating to the renewal theory by the Monte-Carlo method, $\mathrm{Ph}$. D. Thesis, University of Hanoi, 1983.

[2] N. Dunford and J. Schwartz, Linear Operators I, Interscience Publ., New York 1958. 
[3] S. M. Ermakov and V.S. Nefedov, On the estimates of the sum of the Neumann series by the Monte-Carlo method, Dokl. Akad. Nauk SSSR 202 (1) (1972), 27--29 (in Russian).

[4] -, Monte Carlo Method and Related Problems, Nauka, Moscow 1975 (in Russian).

[5] Yu. M. Ermolev, Methods of Stochastic Programming, Nauka, Moscow 1976 (in Russian).

[6] -, Finite Difference Method in Optimal Control Problems, Naukova Dumka, Kiev 1978 (in Russian).

[7] W. H. Fleming and R. W. Rishel, Deterministic and Stochastic Optimal Control, Springer, Berlin 1975.

[8] I. I. Gikhman and A. V. Skorokhod, Introduction to the Theory of Random Processes, Saunders, Philadelphia 1965.

[9] Nguyen Quy $\mathrm{Hy}$, On a probabilistic model for derivation and integration of the solution of some stochastic linear equations, Proc. Conf. Appl. Prob. Stat. Vietnam 1 (10-11), Nhatrang 7-1983.

[10] Nguyen Quy Hy and Nguyen Ngoc Cuong, On probabilistic properties of a solution of a class of random integral equations, Acta Univ. Lodz. Folia Math. 2 (1985).

[11] Nguyen Quy Hy and Nguyen Van Huu, A probabilistic model to solve a problem of stochastic control, Proc. Conf. Math. Vietnam III, Hanoi 7-1985.

[12] Nguyen Quy Hy and Bui Huy Quynh, Solution of a random integral using Monte-Carlo method, Bull. Univ. Hanoi Vol. Math. Mech. 10 (1980).

[13] L. V. Kantorovich and G. P. Akilov, Functional Analysis in Normed Spaces, Gos. Izdat. Fiz.-Mat. Liter., Moscow 1959 (in Russian).

[14] A. I. Khisamutdinov, A unit class of estimates for calculating functionals of solutions to II kind integral equations by the Monte Carlo method, $\mathrm{Zh}$. Vychisl. Mat. i Mat. Fiz. 10 (5) (1970), 1269-1280 (in Russian).

[15] Bui Huy Quynh, On a randomised model for solving stochastic integral equation of the renewal theory, Bull. Univ. Hanoi Vol. Math. Mech. 11 (1980).

FACULTY OF MATHEMATICS, MECHANICS AND INFORMATICS HANOI UNIVERSITY

HANOI, VIETNAM

Reçu par la Rédaction le 30.8.1988

Révisé le 20.7.1989 